\title{
Redundancy Optimization for Series and Parallel Systems Exposed to Random Shocks
}

\author{
Xiaoliang Ling ${ }^{\mathrm{a}, *}$, Yazhou Zhang ${ }^{\mathrm{a}}$, and Ping $\mathrm{Li}^{\mathrm{b}}$ \\ ${ }^{a}$ College of Sciences, Hebei University of Science and Technology, Shijiazhuang, 050018, China \\ ${ }^{b}$ School of Business, Hebei Normal University, Shijiazhuang, 050024, China
}

\begin{abstract}
This paper is devoted to redundancy allocation in series (parallel) systems subject to random shocks. The non-homogeneous Poisson process is used to describe the shock process, and redundant series (parallel) system reliability is given. The majorization order allows for stochastic comparison between random lifetimes of systems under two redundancy allocation policies. Then, the redundancy allocation policy for maximizing (minimizing) the series (parallel) system reliability is presented. Finally, the effect of the number of subsystems on system reliability is analyzed.
\end{abstract}

Keywords: reliability; majorization order; shock model; parallel (series) redundancy

(Submitted on March 10, 2019; Revised on April 16, 2019; Accepted on May 22, 2019)

(C) 2019 Totem Publisher, Inc. All rights reserved.

\section{Introduction}

In reliability engineering, redundancy allocation policy is an effective method of increasing the system reliability. When redundancy allocation policy is used to enhance system reliability, we should evaluate the number of redundancies in each subsystem to satisfy the requirement of system reliability. In general, cold standby redundancy and parallel redundancy are two types of redundancy modes. Since the exact failure time of component is difficult to be observed, the cold standby redundancy is hard to adopt. Therefore, in most cases, parallel redundancy is utilized to enhance system reliability.

Boland et al. [1] studied the issue with respect to allocating redundancy in a system in order to enhance system reliability. Shaked and Shanthikumar [2] considered allocating some parallel redundancies to a series system to achieve the same goal. Singh and Misra [3] studied the assignment of a single parallel redundancy to a series system consisting of two exponential components. They demonstrated that assigning the redundancy to the relatively weaker component can reduce the failure rate of system. $\mathrm{Li}$ and $\mathrm{Hu}$ [4] studied how to assign a parallel redundancy to a series system. They used the stochastic orders theory to compare two series systems with different allocation policies. Misra et al. [5] studied how to allocate several parallel redundancies to a series system. Supposing all redundancies and components are independent and identically distributed (i.i.d.), Hu and Wang [6] considered how to allocate several parallel redundancies to a $k$-out-of- $n$ system. Li and Ding [7] studied how to assign parallel redundancies to a $k$-out-of- $n$ system consisting of nonhomogeneous components. Using the failure rate order, Ding and $\mathrm{Li}[8]$ dealt with how to allocate several parallel redundancies to a $k$ out-of- $n$ system consisting of independent components. Based on different stochastic orders, Valdés et al. [9] compared random lifetimes of series systems with two different redundancy allocation policies. Misra et al. [10] studied stochastic orders relationships between random lifetimes of series (parallel) systems with two different redundancy allocation policies. Li et al. [11] studied a series system consisting of two independent components, and the redundancy allocation policy for maximizing the series system reliability was derived.

All the above papers assume that system reliability is determined by the internal characteristics of the components and that lifetimes of the components are independent. In some situations, however, the system works in a random environment. The influence of the random environment on the system may be described by a shock model. The shock model may stand 
for environmental stress. The stress can result in component failure according to a certain rule. It mainly describes the operating characteristics and failure behavior of a system operating in a shock environment. System failure may be caused by a single large shock or some cumulative shock effect. Based on the different damage effects on the system, shocks can be divided into extreme shocks and cumulative shocks. For models of extreme shocks and cumulative shocks, please refer to Gut and Hüsler [12], Finkelstein [13], Cha and Finkelstein [14-15], Esary et al. [16], Sumita and Shanthikumar [17], Anderson [18], Gut [19], and Montoro-Cazorla and Pérez-Ocón [20].

For a structural system, a shock may result in immediate failure of the system. For example, a powerful earthquake can destroy a bridge with a certain probability or may increase the deterioration of the bridge. In these cases, when the external environment has a large impact, we can ignore the intrinsic characteristics of the components. Component failures may be not independent when a system's failure is caused by an external environment. Some papers have studied the optimal grouping of components policy in a system under a non-homogeneous Poisson shock process. Assuming that the series (parallel) system is exposed to a risk process or combined risk process, Cha [21-22] obtained an optimal components grouping policy. Assuming that a series-parallel phased mission system is subject to shock process, Levitin et al. [23] studied the redundancy allocation policy for maximizing system reliability. In their model, shocks increase the system components failure rate and cause gradual deterioration of the components.

In this paper, the system consists of $k$ components arranged in series (parallel), and for each component, multiple redundancies are assigned to increase (reduce) the system reliability. Random shocks affect all components simultaneously, and component failures are only induced by random shocks. Suppose that a non-homogeneous Poisson process $\{N(t), t \geq 0\}$ is adopted to describe the occurrence of shocks. Denote $\lambda(t)$ as the intensity function of this process. Denote $T_{i}(i=1,2, \cdots)$ as the time at which the $i^{\text {th }}$ shock occurs. Compared with the failure of a component caused by shocks, the failure caused by the intrinsic characteristics of the components themselves can be neglected. Therefore, let components be "absolutely reliable" without shocks. Suppose that the probability of a component's failure is $p(t)$ when each shock occurs at time $t$. Let $q(t)=1-p(t)$ represent the probability that a shock occurring at time $t$ is harmful to the component. In this study, we mainly deal with the redundancy optimization of a series (parallel) system in a shock environment. Sections 2 and 3 give the reliability of a series (parallel) system that is exposed to random shocks and present the optimal redundancy allocation policy.

\section{Notations:}

- $\lambda(t)$

- $k$

- $\boldsymbol{a}=\left(a_{1}, \cdots, a_{k}\right)$

- $m$

- $S(k, \boldsymbol{a})(H(k, \boldsymbol{a}))$

- $\bar{F}_{S(k, \boldsymbol{a})}(t)\left(\bar{F}_{H(k, \boldsymbol{a})}(t)\right)$

- $\mathbb{A}$

- $a=b$

- $\preceq$
The intensity function.

The number of components in the system.

The allocation policy.

The total number of allowed redundancies, $m=\sum_{j=1}^{k} a_{j}$.

The redundant series (parallel) system lifetime with policy $\boldsymbol{a}$.

The reliability function of $S(k, \boldsymbol{a})(H(k, \boldsymbol{a}))$.

The set of all allocation policies $\mathbb{A}=\left\{\left(a_{1}, a_{2}, \cdots, a_{k}\right): m=\sum_{j=1}^{k} a_{j}\right\}$.

This means that $a$ and $b$ have the same sign.

The majorization order.

\section{Series System}

Consider a series system consisting of $k$ components, and suppose that $a_{j}$ redundancies and the $j^{\text {th }}$ component are connected in parallel, $j=1,2, \cdots, k$. Figure 1 gives the redundant structure of the series system.

\subsection{Extreme Shock Model}

The following result presents the reliability function of a redundant series system. 


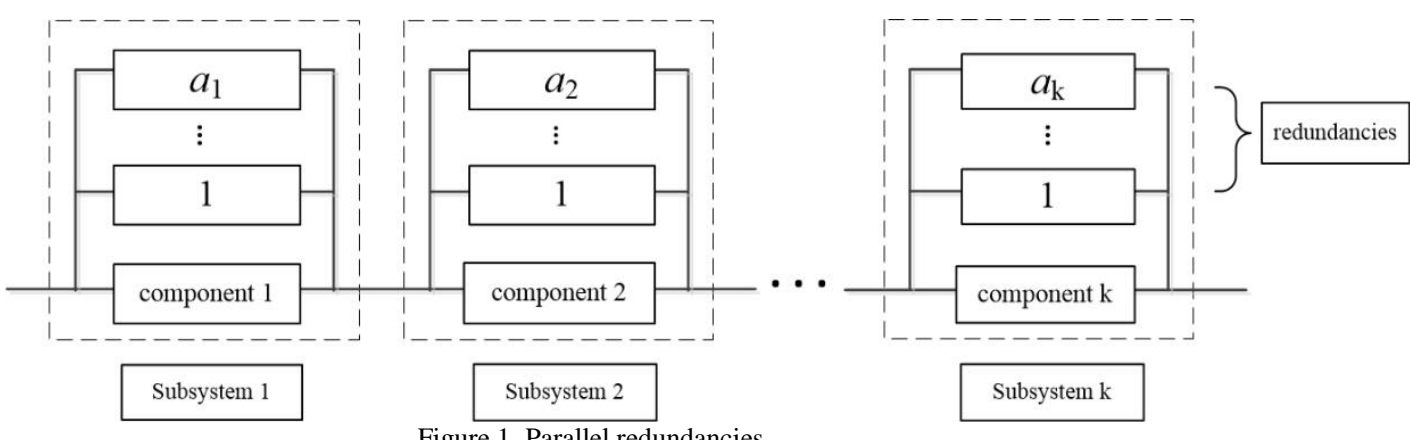

Figure 1. Parallel redundancies

Theorem 2.1 $S(k, a)$ has the reliability function

$$
\bar{F}_{S(k, \boldsymbol{a})}(t)=\sum_{n=0}^{\infty} \int_{0}^{\Lambda(t)} \cdots \int_{0}^{\Lambda(t)} \prod_{j=1}^{k}\left(1-\left(1-\prod_{i=1}^{n}\left(1-p\left(\Lambda^{-1}\left(v_{i}\right)\right)\right)\right)^{a_{j}+1}\right) \mathrm{d} v_{1} \cdots \mathrm{d} v_{n} \frac{\exp \{-\Lambda(t)\}}{n !}
$$

Proof By the double expectation theorem, we have

$$
\bar{F}_{S(k, \boldsymbol{a})}(t)=E\left[\prod_{j=1}^{k}\left(1-\left(1-\prod_{i=1}^{N(t)}\left(1-p\left(T_{i}\right)\right)\right)^{a_{j}+1}\right)\right]
$$

Let $N^{*}(t) \equiv N\left(\Lambda^{-1}(t)\right), t \geq 0$, and $T_{j}^{*} \equiv \Lambda\left(T_{j}\right), j \geq 1$, where $\Lambda(t) \equiv \int_{0}^{t} \lambda(u) \mathrm{d} u$. Then, $\left\{N^{*}(t), t \geq 0\right\}$ is a homogeneous Poisson process with intensity function (1), and $T_{j}^{*}$ is the occurrence time of the $j^{\text {th }}$ shock. Then,

$$
E\left[\prod_{j=1}^{k}\left(1-\left(1-\prod_{i=1}^{N(t)}\left(1-p\left(T_{i}\right)\right)\right)^{a_{j}+1}\right)\right]=E\left[E\left[\prod_{j=1}^{k}\left(1-\left(1-\prod_{i=1}^{N^{*}(s)}\left(1-p\left(T_{i}^{*}\right)\right)\right)^{a_{j}+1}\right) \mid N^{*}(s)\right]\right]
$$

Where $s=\Lambda(t)$. Let random variables $V_{1}, V_{2}, \cdots, V_{n}$ be i.i.d. with common uniform distribution on the interval $[0, \Lambda(t)]$. $\left(V_{(1)}, V_{(2)}, \cdots, V_{(n)}\right)$ are corresponding order statistics. Given $N^{*}(s)=n$, then $\left(T_{1}^{*}, T_{2}^{*}, \cdots, T_{n}^{*}\right)$ and $\left(V_{(1)}, V_{(2)}, \cdots, V_{(n)}\right)$ have the same joint distribution. Since the joint density of $\left(V_{1}, V_{2}, \cdots, V_{n}\right)$ is $1 / \Lambda^{n}(t)$, then

$$
\begin{aligned}
& E\left[\prod_{j=1}^{k}\left(1-\left(1-\prod_{i=1}^{N^{*}(s)}\left(1-p\left(T_{i}^{*}\right)\right)\right)^{a_{j}+1}\right) \mid N^{*}(s)=n\right] \\
= & E\left[\prod_{j=1}^{k}\left(1-\left(1-\prod_{i=1}^{n}\left(1-p\left(\Lambda^{-1}\left(V_{(i)}\right)\right)\right)\right)^{a_{j}+1}\right)\right] \\
= & \int_{0}^{\Lambda(t)} \cdots \int_{0}^{\Lambda(t)} \prod_{j=1}^{k}\left(1-\left(1-\prod_{i=1}^{n}\left(1-p\left(\Lambda^{-1}\left(v_{i}\right)\right)\right)\right)^{a_{j}+1}\right) \frac{1}{\Lambda^{n}(t)} \mathrm{d} v_{1} \cdots \mathrm{d} v_{n}
\end{aligned}
$$

Therefore,

$$
\bar{F}_{S(k, \boldsymbol{a})}(t)=\sum_{n=0}^{\infty} \int_{0}^{\Lambda(t)} \cdots \int_{0}^{\Lambda(t)} \prod_{j=1}^{k}\left(1-\left(1-\prod_{i=1}^{n}\left(1-p\left(\Lambda^{-1}\left(v_{i}\right)\right)\right)\right)^{a_{j}+1}\right) \mathrm{d} v_{1} \cdots \mathrm{d} v_{n} \frac{\exp \{-\Lambda(t)\}}{n !}
$$

Suppose that $p(x) \equiv p, 0 \leq p \leq 1$, and the next result illustrates the effect of $\lambda(t)$ on system reliability. It states that an increase in $\lambda(t)$ will lower the system reliability.

Theorem 2.2 If $\lambda_{1}(t) \leq \lambda_{2}(t)$, then $\bar{F}_{S_{1}(k, a)}(t) \geq \bar{F}_{S_{2}(k, a)}(t)$ for all $t>0$. 
Proof For $i=1,2$, let $N_{i}(t)$ be a non-homogeneous Poisson process having the intensity function $\lambda_{i}(t)$.

It is easy to verify that $P\left(N_{2}(t)=n\right) / P\left(N_{1}(t)=n\right)$ is increasing in $n$. Using Theorem 1.C.1. of Shaked and Shanthikumar [24], we immediately obtain $P\left(N_{1}(t) \geq n\right) \leq P\left(N_{2}(t) \geq n\right)$. It is easy to see that

$$
1-\prod_{j=1}^{k}\left(1-\left(1-\prod_{i=1}^{n}(1-p)\right)^{a_{j}+1}\right)
$$

increases in $n$. Using Equation 1.A.7 of Shaked and Shanthikumar [24], we obtain

$$
E\left[\prod_{j=1}^{k}\left(1-\left(1-\prod_{i=1}^{N_{1}(t)}(1-p)\right)^{a_{j}+1}\right)\right] \geq E\left[\prod_{j=1}^{k}\left(1-\left(1-\prod_{i=1}^{N_{2}(t)}(1-p)\right)^{a_{j}+1}\right)\right]
$$

as the required result.

The diversity of two vectors can be compared in terms of the majorization order (Marshall et al. [25]). Let $\boldsymbol{x}=\left(x_{1}, x_{2}\right.$, $\left.\cdots, x_{n}\right)$ and $\boldsymbol{y}=\left(y_{1}, y_{2}, \cdots, y_{n}\right)$ be two vectors, and $x_{(1)} \geq x_{(2)} \geq \cdots \geq x_{(n)}$ and $y_{(1)} \geq y_{(2)} \geq \cdots \geq y_{(n)}$ be the decreasing arrangement of the elements of $\boldsymbol{x}$ and $\boldsymbol{y}$, respectively. The vector $\boldsymbol{x}$ is said to be majorized by the vector $\boldsymbol{y}$ (denoted by $\boldsymbol{x} \preceq \boldsymbol{y})$ if $\sum_{i=1}^{j} x_{(i)} \leq \sum_{i=1}^{j} y_{(i)}$ and $\sum_{i=1}^{n} x_{(i)}=\sum_{i=1}^{n} y_{(i)}$.

By the majorization order, the next result demonstrates that dividing all redundancies equally can maximize the system reliability.

Theorem 2.3 For $\boldsymbol{a} \in \mathbb{A}$ and $\boldsymbol{a}^{\prime} \in \mathbb{A}$, we have that $\boldsymbol{a} \preceq \boldsymbol{a}^{\prime}$ implies $\bar{F}_{S(k, \boldsymbol{a})}(t) \geq \overline{\boldsymbol{F}}_{S\left(k, \boldsymbol{a}^{\prime}\right)}(t)$.

Proof Let

$$
\phi(\boldsymbol{a})=P\left(S_{(k, \boldsymbol{a})} \leq t\right)=E\left[1-\prod_{j=1}^{k}\left(1-\left(1-\prod_{i=1}^{N(t)}\left(1-p\left(T_{i}\right)\right)\right)^{a_{j}+1}\right)\right], \quad t \geq 0
$$

For $\alpha, \beta=1,2, \cdots, k, \alpha \neq \beta$, we have

$$
\begin{aligned}
& \left(a_{\alpha}-a_{\beta}\right)\left(\frac{\partial \phi(\boldsymbol{a})}{\partial a_{\alpha}}-\frac{\partial \phi(\boldsymbol{a})}{\partial a_{\beta}}\right) \\
\stackrel{\operatorname{sgn}}{=} & \left(a_{\alpha}-a_{\beta}\right) E\left[-\left(1-\prod_{i=1}^{N(t)}\left(1-p\left(T_{i}\right)\right)\right)^{a_{\alpha}+1}\left(1-\left(1-\prod_{i=1}^{N(t)}\left(1-p\left(T_{i}\right)\right)\right)^{a_{\beta}+1}\right)\right. \\
& \left.+\left(1-\prod_{i=1}^{N(t)}\left(1-p\left(T_{i}\right)\right)\right)^{a_{\beta}+1}\left(1-\left(1-\prod_{i=1}^{N(t)}\left(1-p\left(T_{i}\right)\right)\right)^{a_{\alpha}+1}\right)\right] \\
= & \left(a_{\alpha}-a_{\beta}\right) E\left[-\left(1-\prod_{i=1}^{N(t)}\left(1-p\left(T_{i}\right)\right)\right)^{a_{\alpha}+1}+\left(1-\prod_{i=1}^{N(t)}\left(1-p\left(T_{i}\right)\right)\right)^{a_{\beta}+1}\right] \\
\geq & 0
\end{aligned}
$$

Furthermore, it is clear that $\phi(\boldsymbol{a})$ is symmetric with respect to $\boldsymbol{a}=\left(a_{1}, a_{2}, \cdots, a_{k}\right) \in \mathbb{A}$. Then, by Theorem A.4 and Definition A.1 of Marshall et al. [25], we have that if $\boldsymbol{a} \preceq \boldsymbol{a}^{\prime}$, then $\phi(\boldsymbol{a}) \leq \phi\left(\boldsymbol{a}^{\prime}\right)$ as the required result.

Let $\left|a_{j}^{*}-a_{i}^{*}\right| \leq 1, i, j=1,2, \cdots, n, i \neq j$. Using Theorem 4 of Li and Ding [7], we find that $\boldsymbol{a}^{*}=\left(a_{1}^{*}, a_{2}^{*}, \cdots, a_{k}^{*}\right)$ can maximize the series system reliability. 
The next result states that under certain conditions, as the number of subsystems decreases, the system becomes more reliable.

Theorem 2.4 Let $\boldsymbol{a}=\left(a_{1}, a_{2}, \cdots, a_{k}\right)$ and $\boldsymbol{b}=\left(b_{1}, b_{2}, \cdots, b_{k-1}\right)$ satisfy $b_{j} \geq a_{j}$ for any $j=1,2, \cdots, k-1$. We have $\bar{F}_{S(k, a)}(t) \leq \bar{F}_{S(k-1, b)}(t)$.

\subsection{Cumulative Shock Model}

In this section, suppose that the damage caused by shocks is only accumulated. Denote $W\left(T_{j}\right)$ as the damage effect induced by the $j$ th shock. Let $W(t)$ have distribution function $F_{W(t)}(x)$ and density function $f_{W(t)}(x)$. The accumulated damage effect of the system at time $t$ is expressed by

$$
k(t)=\sum_{i=1}^{N(t)} W\left(T_{i}\right)
$$

Assume that the component reaches failure when $k(t)$ is greater than a random boundary $R$. For $l=1,2, \cdots, a_{j}+1$, $j=1,2, \cdots, k$, denote the set of related random variables for component $l$ as

$$
\left\{R_{l} ; W_{l}\left(t_{1}\right), W_{l}\left(t_{2}\right), \cdots, W_{l}\left(t_{N(t)}\right)\right\}
$$

and suppose that random variables $R_{l} \mathrm{~s}$ are i.i.d. and $W_{l}\left(t_{N(t)}\right) \mathrm{s}$ are independent. Suppose that $\{N(t), t \geq 0\}$, $\left\{R_{l}, l=1,2, \cdots, a_{j}+1, j=1,2, \cdots, k\right\}$ and $\left\{W_{l}\left(t_{1}\right), W_{l}\left(t_{2}\right), \cdots, W_{l}\left(t_{N(t)}\right), l=1,2, \cdots, a_{j}+1, j=1,2, \cdots, k\right\}$ are independent. Let $R_{l}$ s have common distribution function $F_{R}(x)$ and density function $f_{R}(x)$. Assume that $f_{R}(x)=v \exp \{-v x\}, x \geq 0$.

Theorem 2.5 $S(k, a)$ has the reliability function

$$
\bar{F}_{S(k, \boldsymbol{a})}(t)=\sum_{n=0}^{\infty} \int_{0}^{\Lambda(t)} \cdots \int_{0}^{\Lambda(t)} \prod_{j=1}^{k}\left(1-\left(1-\prod_{i=1}^{n} \Psi_{W_{1}\left(\Lambda^{-1}\left(v_{i}\right)\right)}(-v)\right)^{a_{j}+1}\right) \mathrm{d} v_{1} \cdots \mathrm{d} v_{n} \frac{\exp \{-\Lambda(t)\}}{n !}
$$

Proof Note that

$$
\begin{aligned}
& P\left(S(k, \boldsymbol{a})>t \mid N(t), T_{1}, T_{2}, \cdots, T_{N(t)}\right) \\
= & \prod_{j=1}^{k}\left(1-P\left(\sum_{i=1}^{N(t)} W_{1}\left(T_{i}\right)>R_{1}, \cdots, \sum_{i=1}^{N(t)} W_{a_{j}+1}\left(T_{i}\right)>R_{a_{j}+1} \mid N(t), T_{1}, T_{2}, \cdots, T_{N(t)}\right)\right)
\end{aligned}
$$

Then,

$$
\begin{aligned}
& P\left(\sum_{i=1}^{N(t)} W_{1}\left(T_{i}\right)>R_{1}, \cdots, \sum_{i=1}^{N(t)} W_{a_{j}+1}\left(T_{i}\right)>R_{a_{j}+1} \mid N(t)=n, T_{1}=t_{1}, T_{2}=t_{2}, \cdots, T_{N(t)}=t_{n}\right) \\
= & \left(P\left(\sum_{i=1}^{n} W_{1}\left(t_{i}\right)>R_{1}\right)\right)^{a_{j}+1} \\
= & \left(\int_{0}^{\infty} \bar{F}_{\sum_{i=1}^{n} W_{1}\left(t_{i}\right)}(x) f_{R}(x) \mathrm{d} x\right)^{a_{j}+1}
\end{aligned}
$$

When $f_{R}(x)=v \exp \{-v x\}, x \geq 0$, we have

$$
\int_{0}^{\infty} \bar{F}_{\sum_{i=1}^{n} W_{1}\left(t_{i}\right)}(x) f_{R}(x) \mathrm{d} x=\int_{0}^{\infty}\left(1-e^{-v x}\right) f_{\sum_{i=1}^{n} W_{1}\left(t_{i}\right)}(x) \mathrm{d} x=1-\Psi_{\sum_{i=1}^{n} W_{1}\left(t_{i}\right)}(-v)
$$


Where $\Psi_{\sum_{i=1}^{n} W_{1}\left(t_{i}\right)}(\cdot)$ is the moments generating function of $\sum_{i=1}^{n} W_{1}\left(t_{i}\right)$. Hence,

$$
\begin{aligned}
\bar{F}_{S(k, \boldsymbol{a})}(t) & =E\left[\prod_{j=1}^{k}\left(1-\left(1-\Psi_{\sum_{i=1}^{N(t)} W_{1}\left(T_{i}\right)}(-v)\right)^{a_{j}+1}\right)\right] \\
& =E\left[E \left[\prod _ { j = 1 } ^ { k } \left(1-\left(1-\Psi_{i=1}^{N(t)} W_{1}\left(T_{i}\right)\right.\right.\right.\right.
\end{aligned}
$$

With a similar proof method of Theorem 2.1, we obtain

$$
\begin{aligned}
& E\left[\prod_{j=1}^{k}\left(1-\left(1-\Psi_{i=1}^{\Psi_{N(t)} W_{1}\left(T_{i}\right)}(-v)\right)^{a_{j}+1}\right) \mid N(t)=n\right] \\
= & \int_{0}^{\Lambda(t)} \cdots \int_{0}^{\Lambda(t)} \prod_{j=1}^{k}\left(1-\left(1-\prod_{i=1}^{n} \Psi_{W_{1}\left(\Lambda^{-1}\left(v_{i}\right)\right)}(-v)\right)^{a_{j}+1}\right) \frac{1}{\Lambda^{n}(t)} \mathrm{d} v_{1} \cdots \mathrm{d} v_{n}
\end{aligned}
$$

Therefore,

$$
\bar{F}_{S(k, \boldsymbol{a})}(t)=\sum_{n=0}^{\infty} \int_{0}^{\Lambda(t)} \cdots \int_{0}^{\Lambda(t)} \prod_{j=1}^{k}\left(1-\left(1-\prod_{i=1}^{n} \Psi_{W_{1}\left(\Lambda^{-1}\left(v_{i}\right)\right)}(-v)\right)^{a_{j}+1}\right) \mathrm{d} v_{1} \cdots \mathrm{d} v_{n} \frac{\exp \{-\Lambda(t)\}}{n !}
$$

By the majorization order, the next result presents that dividing all redundancies equally can maximize the system reliability.

Theorem 2.6 For $\boldsymbol{a} \in \mathbb{A}$ and $\boldsymbol{a}^{\prime} \in \mathbb{A}$, we have that $\boldsymbol{a} \preceq \boldsymbol{a}^{\prime}$ implies $\bar{F}_{S(k, \boldsymbol{a})}(t) \geq \bar{F}_{S\left(k, \boldsymbol{a}^{\prime}\right)}(t)$.

\section{Proof Let}

$$
\phi(\boldsymbol{a})=P(S(k, \boldsymbol{a}) \leq t)=E\left[1-\prod_{j=1}^{k}\left(1-\left(1-\Psi_{\sum_{i=1}^{N(t)} W_{1}\left(T_{i}\right)}(-v)\right)^{a_{j}+1}\right)\right], t \geq 0
$$

For $\alpha, \beta=1,2, \cdots, k, \alpha \neq \beta$, we have

$$
\begin{aligned}
& \left(a_{\alpha}-a_{\beta}\right)\left[\frac{\partial \phi(\boldsymbol{a})}{\partial a_{\alpha}}-\frac{\partial \phi(\boldsymbol{a})}{\partial a_{\beta}}\right]
\end{aligned}
$$

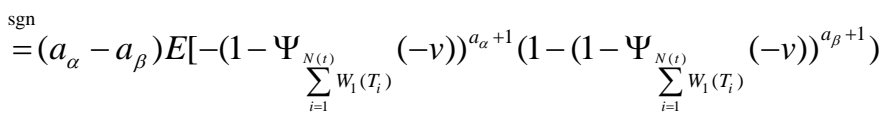

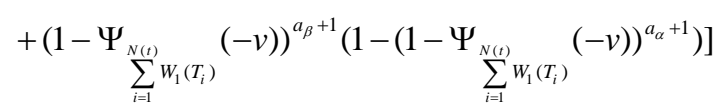

$$
\begin{aligned}
& =\left(a_{\alpha}-a_{\beta}\right) E\left[-\left(1-\Psi_{\sum_{i=1}^{N(t)} W_{1}\left(T_{i}\right)}(-v)\right)^{a_{\alpha}+1}+\left(1-\Psi_{\sum_{i=1}^{N(t)} W_{1}\left(T_{i}\right)}(-v)\right)^{a_{\beta}+1}\right] \\
& \geq 0
\end{aligned}
$$

Furthermore, it is evident that $\phi(\boldsymbol{a})$ is symmetric with respect to $\boldsymbol{a}=\left(a_{1}, a_{2}, \cdots, a_{k}\right) \in \mathbb{A}$. Then, by Theorem A.4 and Definition A.1 of Marshall et al. [25], we have that if $\boldsymbol{a} \preceq \boldsymbol{a}^{\prime}$, then $\phi(\boldsymbol{a}) \leq \phi\left(\boldsymbol{a}^{\prime}\right)$.

The next result states that under certain conditions, as the number of subsystems decreases, the system becomes more reliable. 
Theorem 2.7 Let $\boldsymbol{a}=\left(a_{1}, a_{2}, \cdots, a_{k}\right)$ and $\boldsymbol{b}=\left(b_{1}, b_{2}, \cdots, b_{k-1}\right)$ satisfy $b_{j} \geq a_{j}$ for any $j=1,2, \cdots, k-1$. We have $\bar{F}_{S(k, a)}(t) \leq \bar{F}_{S(k-1, b)}(t)$.

\section{Parallel System}

Consider a parallel system consisting of $k$ components, and suppose that $a_{j}$ redundancies and the $j^{\text {th }}$ component are connected in series, $j=1,2, \cdots, k$. Figure 2 gives the parallel system with series subsystems. For brevity, the following results are given without proof since their proofs are similar to those presented in Section 2.

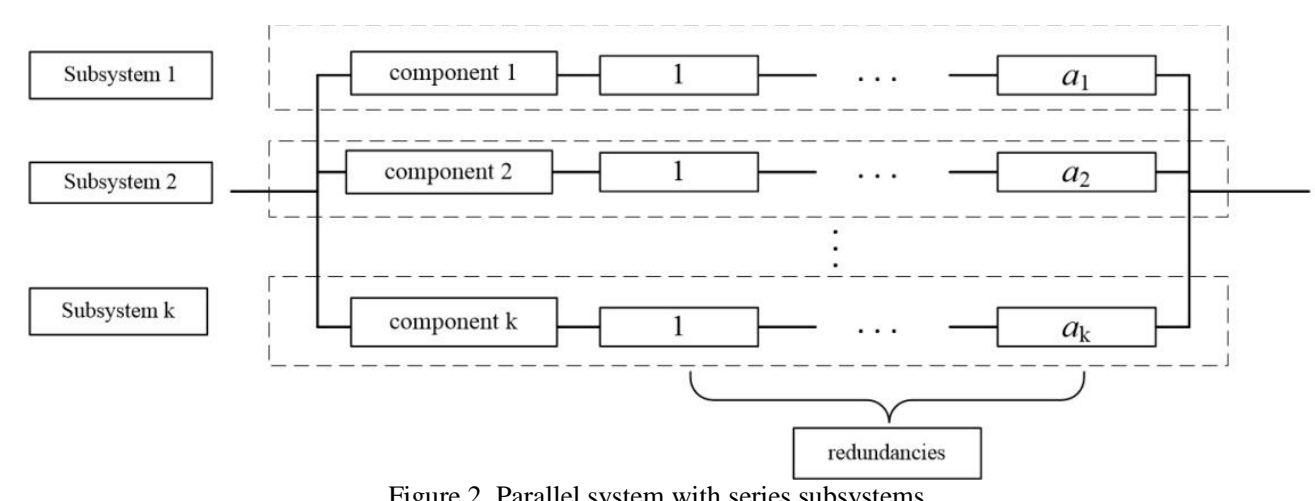

Figure 2. Parallel system with series subsystems

\subsection{Extreme Shock Model}

Theorem 3.1 $H(k, a)$ has the reliability function

$$
\bar{F}_{H(k, a)}(t)=\sum_{n=0}^{\infty} \int_{0}^{\Lambda(t)} \cdots \int_{0}^{\Lambda(t)}\left[1-\prod_{j=1}^{k}\left(1-\left(\prod_{i=1}^{n}\left(1-p\left(\Lambda^{-1}\left(v_{i}\right)\right)\right)\right)^{a_{j}+1}\right] \mathrm{d} v_{1} \cdots \mathrm{d} v_{n} \frac{\exp \{-\Lambda(t)\}}{n !}\right.
$$

Suppose that $p(t) \equiv p, 0 \leq p \leq 1$, and the next result illustrates the effect of $\lambda(t)$ on the system reliability. It states that an increase in $\lambda(t)$ will lower the system reliability.

Theorem 3.2 If $\lambda_{1}(t) \leq \lambda_{2}(t)$, then $\bar{F}_{H_{1}(k, a)}(t) \geq \bar{F}_{H_{2}(k, a)}(t)$ for all $t>0$.

By the majorization order, the next result presents that dividing all redundancies equally can minimize the system reliability.

Theorem 3.3 Let $\boldsymbol{a} \in \mathbb{A}$ and $\boldsymbol{a}^{\prime} \in \mathbb{A}$. If $\stackrel{\mathrm{a}}{\preceq} \boldsymbol{a}^{\prime}$, then $\bar{F}_{H(k, a)}(t) \leq \bar{F}_{H\left(k, \boldsymbol{a}^{\prime}\right)}(t)$.

Let $\left|a_{j}^{*}-a_{i}^{*}\right| \leq 1, i, j=1,2, \cdots, n, i \neq j$. Using Theorem 4 of Li and Ding [7], we have that $\boldsymbol{a}^{*}=\left(a_{1}^{*}, a_{2}^{*}, \cdots, a_{k}^{*}\right)$ can minimize the parallel system reliability.

The next result states that under certain conditions, as the number of subsystems increases, the system becomes more reliable.

Theorem 3.4 Let $\boldsymbol{a}=\left(a_{1}, a_{2}, \cdots, a_{k}\right)$ and $\boldsymbol{b}=\left(b_{1}, b_{2}, \cdots, b_{k-1}\right)$ satisfy $b_{j} \geq a_{j}$ for any $j \in\{1,2, \cdots, k-1\}$. We have $\bar{F}_{H(k-1, b)}(t) \leq \bar{F}_{H(k, a)}(t)$.

\subsection{Cumulative Risks Model}

Like we did in Section 2.2, suppose that the damage induced by shocks is only accumulated. The following result gives the 
reliability function of $H(k, \boldsymbol{a})$ when $f_{R}(x)=v \exp \{-v x\}, x \geq 0$.

Theorem 3.5 $H(k, a)$ has the reliability function

$$
\bar{F}_{H(k, \boldsymbol{a})}(t)=\sum_{n=0}^{\infty} \int_{0}^{\Lambda(t)} \cdots \int_{0}^{\Lambda(t)}\left[1-\prod_{j=1}^{k}\left(1-\left(\prod_{i=1}^{n} \Psi_{W_{1}\left(\Lambda^{-1}\left(v_{i}\right)\right)}(-v)\right)^{a_{j}+1}\right] \mathrm{d} v_{1} \cdots \mathrm{d} v_{n} \frac{\exp \{-\Lambda(t)\}}{n !}\right.
$$

By the majorization order, the next result presents that dividing all redundancies equally can minimize the system reliability.

Theorem 3.6 Let $\boldsymbol{a} \in \mathbb{A}$ and $\boldsymbol{a}^{\prime} \in \mathbb{A}$. If $\boldsymbol{a} \preceq \boldsymbol{a}^{\prime}$, then $\bar{F}_{H(k, a)}(t) \leq \bar{F}_{H\left(k, \boldsymbol{a}^{\prime}\right)}(t)$.

The next result states that under certain conditions, as the number of subsystems increases, the system becomes more reliable.

Theorem 3.7 Let $\boldsymbol{a}=\left(a_{1}, a_{2}, \cdots, a_{k}\right)$ and $\boldsymbol{b}=\left(b_{1}, b_{2}, \cdots, b_{k-1}\right)$ satisfy $b_{j} \geq a_{j}$ for any $j=1,2, \cdots, k-1$. We have $\bar{F}_{H(k-1, b)}(t) \leq \bar{F}_{H(k, \boldsymbol{a})}(t)$.

\section{Conclusions}

In order to increase (decrease) the system reliability of a series (parallel) system working in a shock environment, some redundancies are assigned to the system. This paper mainly studies the components assignment problem of series (parallel) systems exposed to shock environments. Extreme shock and cumulative shock are used to describe the shock environment. We obtain the redundant system reliability and compare reliability functions of redundant systems with different shock intensity functions. Then, we give the optimal allocation strategy for series (parallel) systems in shock environments.

\section{Acknowledgments}

This work was supported by the Young Talent Support Plan of Hebei Province, the Foundation of Hebei Education Department (BJ2016021, QN2018129), the National Natural Science Foundation of China (11501162), and the Science Foundation of Hebei Normal University (L2017B24).

\section{References}

1. P. J. Boland, E. El-Neweihi, and F. Proschan, "Stochastic Order for Redundancy Allocation in Series and Parallel Systems," Advances in Applied Probability, Vol. 24, No. 1, pp. 161-171, 1992

2. M. Shaked and J. G. Shanthikumar, "Optimal Allocation of Resources to Nodes of Parallel and Series Systems," Advances in Applied Probability, Vol. 24, No. 4, pp. 894-914, 1992

3. H. Singh and N. Misra, "On Redundancy Allocations in Systems," Journal of Applied Probability, Vol. 31, No. 4, pp. 10041014, 1994

4. X. Li and X. Hu, "Some New Stochastic Comparisons for Redundancy Allocations in Series and Parallel Systems," Statistics and Probability Letters, Vol. 78, No. 18, pp. 3388-3394, 2008

5. N. Misra, I. D. Dhariyal, and N. Gupta, "Optimal Allocation of Active Spares in Series Systems and Comparison of Component and System Redundancies," Journal of Applied Probability, Vol. 46, No. 1, pp. 19-34, 2009

6. T. Hu and Y. Wang, "Optimal Allocation of Active Redundancies in r-out-of-n Systems," Journal of Statistical Planning and Inference, Vol. 139, pp. 3733-3737, 2009

7. X. Li and W. Ding, "Optimal Allocation of Active Redundancies to k-out-of-n Systems with Heterogeneous Components," Journal of Applied Probability, Vol. 47, No. 1, pp. 254-263, 2010

8. W. Ding and X. Li, "The Optimal Allocation of Active Redundancies to k-out-of-n Systems with Respect to Hazard Rate Ordering," Journal of Statistical Planning and Inference, Vol. 142, No. 7, pp. 1878-1887, 2012

9. J. E. Valdés, G. Arango, R. I. Zequeira, and G. Brito, "Some Stochastic Comparisons in Series Systems with Active Redundancy," Statistics and Probability Letters, Vol. 79, No. 11, pp. 945-949, 2010

10. N. Misra, A. K. Misra, and I. D. Dhariyal, "Standby Redundancy Allocations in Series and Parallel Systems," Journal of Applied Probability, Vol. 48, No. 1, pp. 43-55, 2011

11. X. Li, Y. Wu, and Z. Zhang, "On the Allocation of General Standby Redundancy to Series and Parallel Systems," Communications in Statistics, Vol. 42, No. 22, pp. 4056-4069, 2013

12. A. Gut and J. Hüsler, "Extreme Shock Models," Extremes, Vol. 2, No. 3, pp. 295-307, 1999 
13. M. S. Finkelstein, "Simple Bounds for Terminating Poisson and Renewal Shock Processes," Journal of Statistical Planning and Inference, Vol. 113, No. 2, pp. 541-549, 2003

14. J. H. Cha and M. S. Finkelstein, "On a Terminating Shock Process with Independent Wear Increments," Journal of Applied Probability, Vol. 46, No. 2, pp. 353-362, 2009

15. J. H. Cha and M. S. Finkelstein, "On New Classes of Extreme Shock Models and Some Generalizations," Journal of Applied Probability, Vol. 48, No. 1, pp. 258-270, 2011

16. J. D. Esary, A. W. Marshall, and F. Proschan, "Shock Models and Wear Processes," Annals of Probability, Vol. 1, No. 4, pp. $627-649,1973$

17. U. Sumita and J. G. Shanthikumar, "A Class of Correlated Cumulative Shock Models," Advances in Applied Probability, Vol. 17, No. 2, pp. 347-366, 1985

18. K. K. Anderson, “A Note on Cumulative Shock Models,” Journal of Applied Probability, Vol. 25, No. 1, pp. 220-223, 1988

19. A. Gut, "Cumulative Shock Models," Advances in Applied Probability, Vol. 22, No. 2, pp. 504-507, 1990

20. D. Montoro-Cazorla and R. Pérez-Ocón, "A Reliability System under Cumulative Shocks Governed by a BMAP," Applied Mathematical Modelling, Vol. 39, No. 23-24, pp. 7620-7629, 2015

21. J. H. Cha, "Comparison of Combined Stochastic Risk Processes and its Applications," European Journal of Operational Research, Vol. 215, No. 2, pp. 404-410, 2011

22. J. H. Cha, "Stochastic Comparison of Generalized Combined Risk Processes," Journal of Statistical Planning Inference, Vol. 143, No. 4, pp. 818-826, 2013

23. G. Levitin, M. Finkelstein, and Y. Dai, "Redundancy Optimization for Series-Parallel Phased Mission Systems Exposed to Random Shocks," Reliability Engineering and System Safety, Vol. 167, pp. 554-560, 2017

24. M. Shaked and J. G. Shanthikumar, "Stochastic Orders," Springer, New York, 2007

25. A. W. Marshall, I. Olkin, and B. C. Arnold, "Inequalities: Theory of Majorization and its Applications," Springer, New York, 2011 